ORIGINAL ARTICLE

\title{
Can urine dipstick testing for urinary tract infection at point of care reduce laboratory workload?
}

\author{
H D Patel, S A Livsey, R A Swann, S S Bukhari
}

J Clin Pathol 2005;58:951-954. doi: 10.1136/icp.2004.025429

\begin{abstract}
Aim: The University Hospitals of Leicester NHS Trust microbiology laboratory receives 150000 urine samples each year, approximately $80 \%$ of which prove to be culture negative. The aim of this study was to reduce the proportion of culture negative urines arriving in the laboratory, by producing local evidence based guidelines for the use of urine dipstick testing at point of care within the trust's three acute hospitals. Methods: One thousand and seventy six unborated urine samples were dipstick tested at the point of care using an automatic strip reader. Quantitative results for the four infection associated markers-leucocyte esterase, nitrite, blood, and protein-were compared with the results of conventional laboratory microscopy and culture.

Results: The performance of different marker combinations was calculated against the routine laboratory methods. One hundred and seventy five (16.3\%) samples were negative for all four markers. Of these dipstick negative samples, only three (1.7\% of all true positives) were positive by culture. The absence of all four infection associated markers was found to have a greater than $98 \%$ negative predictive value and a sensitivity and specificity of $98.3 \%$ and $19.2 \%$, respectively.

Conclusions: A urinary dipstick testing algorithm for infection associated markers was derived for use in hospital patients to screen out negative urines. Two years after distributing the algorithm and promoting access to reagent strips and strip readers, a reduction in the urine workload has been seen against an otherwise increasing laboratory specimen load.
\end{abstract}

See end of article for authors' affiliations .....................

Correspondence to: Dr S S Bukhari,

Sandringham Building, Floor 5, Leicester Royal Infirmary, Infirmary

Square, Leicester LE1 5WW, UK; sayed.bukhari@ uhl-tr.nhs.uk

Accepted for publication 14 January 2005
U rinary tract infection (UTI) is one of the most common clinical conditions in both hospital and the community. Almost a third of all specimens sent to our laboratory are requests for the investigation of possible UTI. Of a total of 150000 urine samples received each year, approximately one third originate from within the hospital trust. Overall, $80 \%$ of urine samples are found to be negative for bacteriuria. Laboratory resources and patient care could be improved by the appropriate use of a validated near patient test for UTI.

\section{"Almost a third of all specimens sent to our laboratory are requests for the investigation of possible urinary tract infection"}

The role of biochemical markers in screening for urinary tract infections in the near patient setting is well established. ${ }^{2}$ Studies have concluded that near patient urine dipstick testing (NPUDT) is a more accurate method for diagnosing UTI than clinical assessment on its own. ${ }^{3}$ In addition, NPUDT is better at excluding UTI when the results are negative than confirming a diagnosis of UTI when they are positive. ${ }^{4}$ This methodology is also simple and relatively inexpensive. The four infection associated biochemical markers have been shown to have a negative predictive value of $97-99 \% .^{12}$

In an attempt to promote the use of reagent strip testing as point of care infection screening, the performance of NPUDT was compared with conventional laboratory techniques in the local setting. Because the efficacy of dipstick testing may vary with different patient populations, urine samples from hospital inpatients were examined initially.

\section{METHODS}

To facilitate this programme, the Leicester laboratory established a multidisciplinary group consisting of medical, nursing, technical, and pharmacy representatives. The study plan was to undertake near patient testing of unborated urine samples in suspected cases of UTI, using reagent strip tests (Multistix 8SG; Bayer Diagnostics, Newbury, Berkshire, UK) and a strip reader (Clinitek 50; Bayer Diagnostics). The use of a Clinitek reader has been shown to increase the sensitivity of the strip tests by reducing observer error. ${ }^{5}$ The participants were asked to attach paper printouts from the readers to a conventional completed microbiology request form and send these to the laboratory with the urine sample, after transferring it into a boric acid container. All samples were processed on the day of arrival, including weekends.

The University Hospitals of Leicester NHS Trust is composed of three acute hospitals, namely: Leicester Royal Infirmary (LRI), Leicester General Hospital (LGH), and Glenfield General Hospital (GGH). All three sites use a single microbiology service. During the trial, the limited availability of dipstick readers required us to select participating wards. The main users from each of the hospital sites were selected. We aimed to obtain at least 1000 data sets before analysing the results.

Laboratory tests consisted of inverted microscopy to provide semiquantitative counts of erythrocytes, leucocytes, and epithelial cells. This was performed as part of our current routine protocol. Calibrated loops were used to culture $1 \mu \mathrm{l}$ of urine on to quarter CLED plates. These plates were incubated aerobically at $37^{\circ} \mathrm{C}$ overnight. Culture was used as the reference method for determining the performance of the dipstick data.

Any samples indicating a trace or more of leucocyte esterase, nitrite, blood, or protein were considered positive

Abbreviations: CFU, colony forming units; GGH, Glenfield General Hospital; LGH, Leicester General Hospital; LRI, Leicester Royal Infirmary; and NPUDT, near patient urine dipstick testing; NPV, negative predictive value; UTI, urinary tract infection 
Table 1 Urine screening using different infection associated marker combinations

\begin{tabular}{llllll}
\hline $\begin{array}{l}\text { Marker } \\
\text { combination }\end{array}$ & $\begin{array}{l}\text { Significant } \\
\text { cultures missed }\end{array}$ & $\begin{array}{l}\text { Percentage screened } \\
\text { out }\end{array}$ & NPV & Sensitivity (\%) & Specificity (\%) \\
\hline L/N/B/P & 3 & 16.3 & 98.3 & 98.3 & 19.2 \\
L/B/P & 4 & 16.8 & 97.8 & 97.8 & 19.8 \\
L/N/B & 5 & 25.7 & 98.2 & 97.2 & 30.2 \\
L/N/P & 6 & 28.2 & 98.0 & 96.7 & 33.1 \\
N/B/P & 18 & 27.9 & 94.0 & 90.0 & 31.5 \\
L/N & 17 & 48.0 & 96.7 & 90.6 & 55.8 \\
\hline
\end{tabular}

Sensitivity = true positives/(true positives+false negatives) $\times 100$; specificity $=$ true negatives $/$ (true negatives + false positives) $\times 100$; negative predictive value $(N P V)=$ true negative/(true negative+false negative) $\times 100$.

$B$, blood; L, leucocyte esterase; N, nitrite; P, protein.

for dipstick testing. The criterion for clinically significant bacteriuria was either a pure or predominant culture of $>10^{5}$ colony forming units $(\mathrm{CFU}) / \mathrm{ml}$, two organisms in similar proportions at $>10^{5} \mathrm{CFU} / \mathrm{ml}$, or $10^{4}-10^{5} \mathrm{CFU} / \mathrm{ml}$ of a Gram negative organism or two organisms where the Gram negative clearly predominates (PHLS Standard Operating Procedures. Investigation of Urine. B.SOP 4l Issue no l, issue date 02.04.1998. Issued by technical services, PHLS Headquarters) ${ }^{6}$

\section{RESULTS}

In total, 1076 comparative data sets were collected and analysed using combinations of infection associated markers. One hundred and seventy five (16.3\%) samples were negative for all four markers and 180 samples were positive for a significant culture. Table 1 shows the performance of these various marker combinations.

The results indicate that the laboratory urine workload could be reduced by at least $16 \%$ through the introduction of the most cautious dipstick testing; that is, all four markers being negative as the screening criteria. This could be increased to over $28 \%$ if the presence of blood is ignored and $48 \%$ if leucocyte esterase and nitrite only are used as the screening criteria. Using these criteria, three $(1.7 \%$ of all true positives), six (3.3\%), and $17(9.4 \%)$ significant cultures would be missed, respectively.

The group recommended an algorithm that had the minimum effect on the quality of service that was provided but would allow a considerable reduction in the number of negative urines received.

On this basis it was decided to initiate a screening programme based on all four infection associated markers. An algorithm was designed, colourfully presented, copied, and laminated. Copies of the algorithm were distributed to all wards and clinics, and remain in use (fig 1).

The number of urine samples received a year before the distribution of the algorithm was examined, to provide information on the impact of the algorithm on urine workload (table 2).
The impact of dipstick screening on the percentage of positive urines for each of the two years after the distribution of the algorithm was examined (table 3 ).

\section{DISCUSSION}

Laboratories are constantly trying to provide a high quality service. The quality review should encompass preanalytical aspects, including advice to users on appropriate specimen collection and point of care testing.

The aim of our study was not just to confirm evidence already available about the benefits of using NPUDT but also to encourage the routine use of dipstick testing to screen out the negative urine workload and evaluate its impact on laboratory activity.

The process involved establishing a dynamic multidisciplinary team to make decisions focused on implementing the use of NPUDT. From the outset the team determined methods of providing and obtaining constant feedback during and after the study. Initially, this was in the form of formal letters, followed by on site support in terms of training in reagent handling and instrument use. The training and use of dipstick testing was well received by users. To identify and focus on any problem areas that might arise, laboratory workload data were initially reviewed monthly for each of the three acute hospitals.

Various studies have examined patient populations separately. These have predominantly focused on the elderly ${ }^{58}$ or children, ${ }^{9-13}$ and have used different combinations of biochemical tests and appearance of the urine in their screening methods. Studies have examined specific groups of patients, such as urological patients, where dipstick testing has been used to select patients for perioperative antibiotics. ${ }^{14}$ Although we have examined our data using criteria such as age, ward origin, and sex, we summarised our results as shown here.

Table 1 shows the performance of six different infection associated marker combinations. A study using nitrite and leucocyte esterase to detect UTIs showed nitrite to be more reliable. ${ }^{15}$ Another advocated a double marker screening approach using nitrite and leucocyte detection, ${ }^{5}$ whereas

Table 2 Urine workload before and after the introduction of the urine dipstick algorithm for each hospital site.

\begin{tabular}{llll}
\hline Hospital site & $\begin{array}{l}\text { One year before algorithm } \\
\text { introduction }\end{array}$ & $\begin{array}{l}\text { One year after algorithm } \\
\text { distribution (\% change) }\end{array}$ & $\begin{array}{l}\text { Two years after algorithm } \\
\text { distribution (\% change) }\end{array}$ \\
\hline LRI & 21267 & $-237(-1.1)$ & $-997(-4.7)$ \\
LGH & 22065 & $-2333(-10.6)$ & $-1917(-8.7)$ \\
GGH & 6571 & $+557(+8.8)$ & $+2311(+35.2)$ \\
Total & 49903 & $-1993(-4.0)$ & $-603(-1.2)$ \\
LRI and LGH & 43332 & $-2570(-5.9)$ & $-2914(-6.7)$ \\
\hline
\end{tabular}

Each year is compared with the year before the distribution of the algorithm.

GGH, Glenfield General Hospital; LGH, Leicester General Hospital; LRI, Leicester Royal Infirmary. 


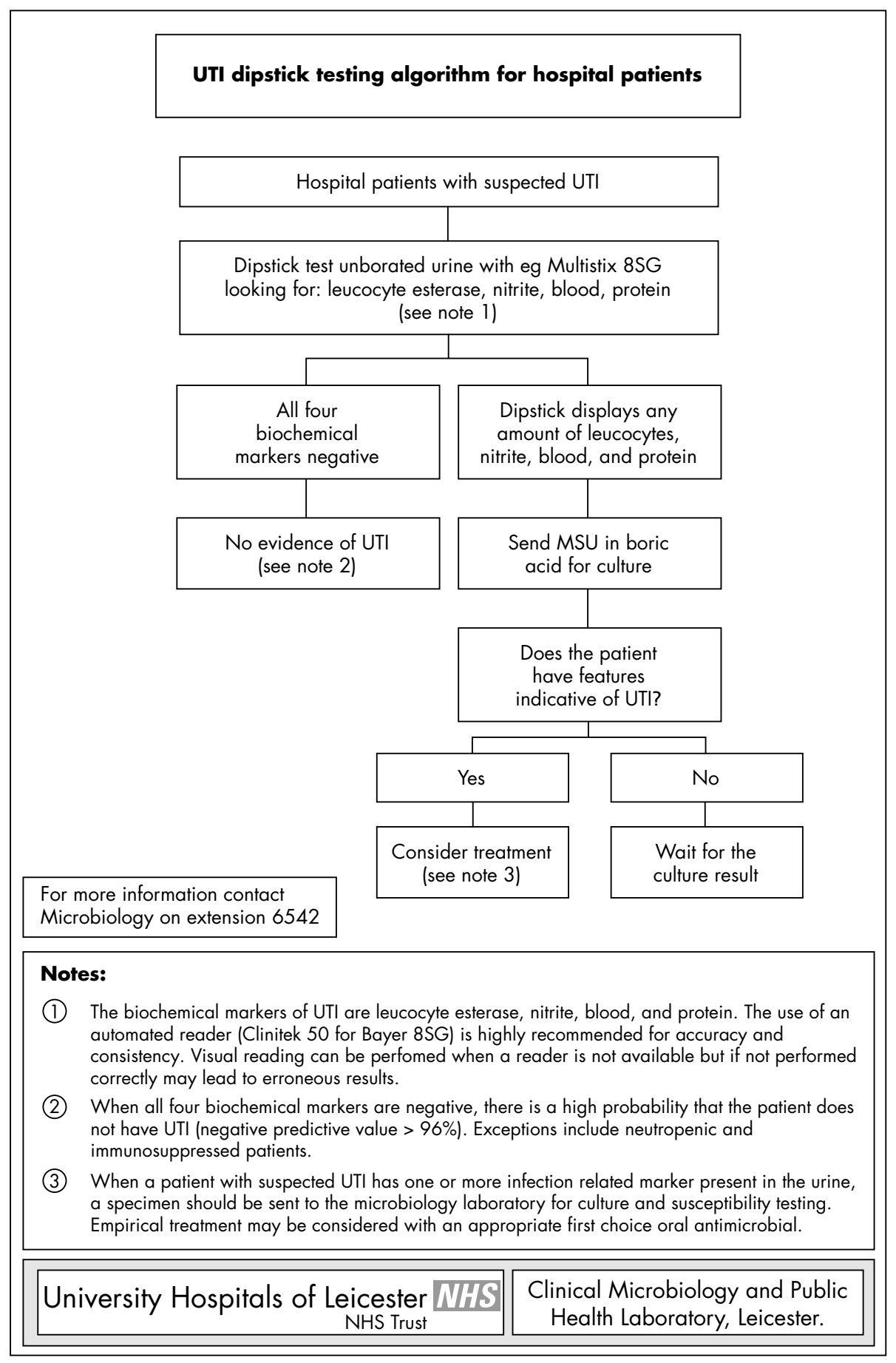

Figure 1 Urine dipstick testing algorithm distributed to wards, August 2001. MSU, midstream urine; UTI, urinary tract infection.

other workers recommend the use of a combination of three infection associated markers (nitrite, protein, and blood), which gives a negative predictive value (NPV) of over $98 \% .{ }^{16}$ In general, the fewer markers that are tested, the greater the proportion of specimens screened out. Our data showed that a leucocyte esterase and nitrite combination screened out approximately half of all urines while retaining a respectable NPV of $96.7 \%$ and a $5 \%$ false negative rate. This degree of false negativity is much lower than that experienced by Zaman et al, ${ }^{17}$ who do not recommend using these two markers for screening hospital inpatients.

The study group advocated the use of all four infection associated markers at any level of positivity to achieve the maximum NPV of $98.3 \%$ and a resulting sensitivity and specificity of $98.3 \%$ and $19.2 \%$, respectively, demonstrating a classic loss in specificity for gains in sensitivity.

Table 2 presents the change in urine workload for the two years after the distribution of the algorithm. The first year after the algorithm had been distributed showed a marginal decrease in urine workload for the LRI site, a significant decrease at the LGH site, and an increase at the GGH site. The urine workload continued to decrease for the second year at the LRI and LGH sites but significantly increased at the GGH site for the reasons discussed below.

The overall reduction in urine workload was less than that anticipated. From the outset, this study lacked representation 
Table 3 Percentage change in positivity in the first and second years after the introduction of the urine dipstick algorithm

\begin{tabular}{lll}
\hline Hospital site & $\begin{array}{l}\text { First year after algorithm } \\
\text { distribution }\end{array}$ & $\begin{array}{l}\text { Second year after } \\
\text { algorithm distribution }\end{array}$ \\
\hline LRI & +1.7 & +2.7 \\
LGH & +3.5 & +4.5 \\
GGH & +2.6 & +1.2 \\
Total & +2.7 & +3.4 \\
LRI and LGH & +2.8 & +3.7 \\
\hline
\end{tabular}

Each year is compared with the year before the distribution of the algorithm.

GGH, Glenfield General Hospital; LGH, Leicester General Hospital; LRI, Leicester Royal Infirmary.

from GGH. Initially, this was not considered to be a major problem, because only $15 \%$ of the total inpatient urines were received from GGH, with few major identifiable users. A possible reason for this pronounced increase in urine workload for GGH may be that on completion of the trial and during the distribution of the algorithm, the three hospital sites had been managed separately, but subsequently were amalgamated to form the University Hospitals of Leicester NHS Trust, with a considerable reconfiguration of the clinical services provided at the three sites.

It is impossible to identify the precise contribution of this study to the reduction in urine workload. Examination of the overall clinical bacteriology workload has shown an annual increase. However it would appear that the urine workload has been kept in check through the promotion of dipstick testing. This is supported by the data presented in table 3 , which demonstrate a steady increase in the positivity rate over the two years after implementation of the algorithm.

The "urine dipstick study group" meets regularly to monitor the urine workload. We have endeavoured to maintain a broad programme of education, including discussions with groups on the benefits of dipstick testing, demonstrations on the use of reagent strip readers, and the introduction of the algorithms to the University Hospitals of Leicester user handbook.

"The advantage of near patient urine dipstick testing for general practitioners is that decisions on antimicrobial prescribing or patient reassurance can be made immediately"

Controlled trials have been recommended to examine the outcomes, costs of care, and patient satisfaction from different diagnostic and management strategies. ${ }^{18}$

Our confidence in the results and the approach adopted to manage the number of inpatient urines received by our laboratory led us to initiate a similar study and subsequent development of an algorithm for urines sent by general practitioners. Previous studies have shown an NPV of $98.5 \%$ and a potential reduction in workload of $40 \%$ for this group. ${ }^{19}$ The advantage of NPUDT for general practitioners is that decisions on antimicrobial prescribing or patient reassurance can be made immediately. We plan to ascertain the impact of a similar algorithm on urine workload in primary care.

Urinary dipstick testing for UTI in the near patient setting is a valuable resource to screen out negative urine specimens at the point of care. If properly implemented this programme
Take home messages

- We derived a urinary dipstick testing algorithm for infection associated markers for use in hospital patients to screen out negative urines

- The absence of all four infection associated markers (blood, leucocyte esterase, nitrite, and protein) had a greater than $98 \%$ negative predictive value and a sensitivity and specificity of $98.3 \%$ and $19.2 \%$, respectively

- Two years after distributing the algorithm and promoting access to reagent strips and strip readers, a reduction in the urine workload has been seen against an otherwise increasing laboratory specimen load

- Work is in progress to extend this algorithm to primary care, where even greater savings could be made

can result in improved use of laboratory resources and can aid clinicians in instant clinical decision making.

\section{Authors' affiliations}

H D Patel, S A Livsey, R A Swann, S S Bukhari, Department of Clinical Microbiology, University Hospitals of Leicester NHS Trust, Leicester LEI 5WW, UK

\section{REFERENCES}

1 Barker BA, Ratcliffe JG, Turner GC. Urine screening for leucocytes and bacteria by dipstick and reflectance spectrophotometry. Med Lab Sci 1989:46:97-100.

2 Stevens M. Screening urines for bacteriuria. Med Lab Sci 1989;46:194-206.

3 Sultana RV, Zalstein S, Cameron P, et al. Dipstick urinalysis and the accuracy of the clinical diagnosis of urinary tract infection. J Emerg Med 2001;20:13-19.

4 Fowlis GA, Waters J, William G. The cost effectiveness of combined rapid tests (Multistix) in screening for urinary tract infections. J R Soc Med 1995;88:303.

5 Fulcher RA, Maisey SP. Evaluation of dipstick tests and reflectance meter for screening for bacteriuria in elderly patients. Br J Clin Pract 1991;45:245-6.

6 Kass EH. Asymptomatic infections of the urinary tract. Trans Assoc Am Physicians 1956;61:56-64.

7 Flanagan PG, Davies EA, Rooney PG, et al. Evaluation of four screening tests for bacteriuria in elderly people. Lancet 1989;1:1117-19.

8 McNaughton $M$, Cavanagh S. Changing urine testing protocols through audit. Nurs Times 1998;94:42-3.

9 Wiggelinkhuizen J, Maytham D, Hanslo DH. Dipstick screening for urinary tract infection. S Afr Med J 1988;74:224-8.

10 Lejeune B, Baron R, Guillois B, et al. Evaluation of a screening test for detecting urinary tract infection in newborns and infants. J Clin Pathol 1991;44:1029-30.

11 Woodward MN, Griffiths DM. Use of dipsticks for routine analysis of urine from children with acute abdominal pain. BMJ 1993;306:1512.

12 Molyneux EM, Robson WJ. A dipstick test for urinary tract infections. J Accid Emerg Med 1995;12:191-3.

13 Sharief N, Hameeed M, Petts D. Use of rapid dipstick tests to exclude urinary tract infection in children. Br J Biomed Sci 1998;55:242-6.

14 Mills SJ, Ford M, Gould FK, et al. Screening for bacteriuria in urological patients using reagent strips. Br J Urol 1992;70:314-17.

15 Ravichandran D, Daltrey I, Uglow $M$, et al. Urine testing for acute lower abdominal pain in adults. Br J Surg 1994;81:1460-1.

16 Lowe PA. Chemical screening and prediction of bacteriuria-a new approach. Med Lab Sci 1986;43:28-33.

17 Zaman Z, Borremans A, Verhaegen J, et al. Disappointing dipstick screening for urinary tract infection in hospital inpatients. J Clin Pathol 1998;51:471-2.

18 Bent S, Saint S. The optimal use of diagnostic testing in women with acute uncomplicated cystitis. Am J Med 2002;113:20-8.

19 Hiscoke C, Yoxall H, Greig D, et al. Validation of a method for the rapid diagnosis of urinary tract infection suitable for use in general practice. Br J Gen Pract 1990;40:403-5. 